\title{
Propagación vegetativa de Berberidopsis corallina Hook.f., una especie en peligro de extinción, endémica de Chile
}

\section{Vegetative propagation of Berberidopsis corallina Hook.f., an endangered species, endemic to Chile}

\author{
Matilde E. Uribe ${ }^{1,2 *}$, Ricardo Durán 1 , Gastón Bravo ${ }^{1}$, Freddy Mora ${ }^{1}$, Priscila Cartes ${ }^{1}$, Catherine \\ DeLAVEAU ${ }^{1,2}$
}

1'Departamento de Silvicultura, Facultad de Ciencias Forestales, Universidad de Concepción, Victoria 631, Barrio Universitario, Casilla 160-C, Concepción, Chile.

${ }^{2}$ Centro de Biotecnología-Laboratorio Cultivo de Tejidos, Universidad de Concepción, Barrio Universitario s/n, Concepción, Chile.

*muribe@udec.cl

\begin{abstract}
RESUMEN
Berberidopsis corallina es una planta trepadora endémica de Chile catalogada "en peligro de extinción" y que hoy se encuentra en problemas de conservación, debido al escaso número de poblaciones y a su difícil reproducción por semillas. El objetivo del presente trabajo fue evaluar su capacidad de enraizamiento de estacas recolectadas en distintas épocas del año y utilizando diferentes concentraciones de la auxina ácido 3-indolbutírico. Las muestras se obtuvieron de plantas madres que crecen en Trongol Bajo (Curanilahue 37⒉ S; $73^{\circ} 20^{\prime} \mathrm{O}$, provincia de Arauco) en época de verano, otoño e invierno de 2006. Estacas de 8-10 cm de longitud fueron tratadas bajo inmersión rápida a diferentes concentraciones de ácido 3-indolbutírico $(0,500,1.500,3.000,5.000$ ppm). Después de cinco meses de realizado el ensayo se evaluó el efecto del ácido 3-indolbutírico (AIB) y época de muestreo sobre el potencial de enraizamiento adventicio en las estacas. El estudio reveló que existen diferencias significativas en cuanto a la época de recolecta, alcanzándose un mayor porcentaje de enraizamiento en estacas recolectadas en invierno (93\%); sin embargo, entre los tratamientos no existen diferencias significativas. El mayor número de raíces se obtuvo en el tratamiento 500 ppm con un valor promedio de 8,1 raíces por estaca en época de otoño. Se concluye que $B$. corallina puede ser reproducido vegetativamente sin la necesidad de aplicar auxina a la estaca en época de invierno y otoño, en plantas procedentes de Trongol Bajo, permitiendo propagar la especie con la mínima intervención.
\end{abstract}

Palabras clave: Ácido 3-indolbutírico, Berberidopsis corallina, enraizamiento adventicio, época de colecta.

\begin{abstract}
Berberidopsis corallina is an endangered vine endemic to Chile, which is difficult to conserve due to its small number of remaining populations and its recalcitrance to reproduce by seeds. The objective of this study was to evaluate the rooting ability of cuttings collected at different times and treated with varying concentrations of the auxin indole3-butyric acid. Semi-ligneous cuttings were collected from plant material found in Trongol Low (Curanilahue $37^{\circ}$ $28^{\prime} \mathrm{S}, 73^{\circ} 20^{\prime} \mathrm{W}$, Arauco province) during the summer, autumn and winter of 2006. Cuttings of $8-10 \mathrm{~cm}$ in length were rapidly immersed in solutions with different concentrations of indole-3-butyric acid $(0,500,1,500,3,000,5,000$ $\mathrm{ppm}$ ). The effect of indole-3-butyric acid (IBA) and collection time of the cuttings on their adventitious rooting potential were evaluated five months after rapid immersion. Significant differences existed with regard to the time of collection, with a higher percentage of rooting observed in cuttings collected in winter (93\%); however, between the treatments there were no significant differences. The highest number of roots was obtained when autumn cuttings were treated with $500 \mathrm{ppm}$ IBA, yielding an average of 8.1 roots per cutting. The authors conclude that $B$. corallina can be vegetatively reproduced via cuttings without exogenous hormone addition if the cuttings are selected during winter and autumn from plants in Trongol Low, which allows for the efficient propagation of this endangered species.
\end{abstract}

KEYWORDs: Indole-3-butyric acid, Berberidopsis corallina, adventitious rooting, collection time. 


\section{INTRODUCCIÓN}

En el bosque nativo chileno se encuentran numerosas especies dentro de alguna categoría de amenaza, incluso algunas de ellas desaparecen antes de conocer sus características y variación genética (Hechenleitner et al. 2005). Dentro de la categoría "en peligro de extinción" se encuentra la trepadora endémica Berberidopsis corallina Hook.f. (michay rojo) (Benoit 1989), que además según las categorías de la UICN Versión 3.1, fue catalogada como una especie "en peligro" (Hechenleitner et al. 2005). Michay rojo forma parte de los bosques laurifolios de la vertiente occidental de la Cordillera de la Costa entre la VII (provincia de Cauquenes) y X regiones (provincia de Llanquihue) (Smith-Ramírez et al. 2005). Recientemente fueron descubiertas nuevas poblaciones en 65 fragmentos de vegetación y bosques nativos con fines de protección, al interior de predios de la empresa forestal Bosques Arauco S.A., cuyos registros incrementan significativamente la distribución espacial y altitudinal conocida para esta especie (Alarcón et al. 2007). En conjunto con otras especies protegidas caracteriza el sector costero del extremo sudoccidental de la VII Región del Maule, donde se ha desarrollado una vegetación nativa, cuya importancia radica en el hecho de estar constituida por especies de interés biogeográfico y taxonómico, así como para la conservación y ecoturismo (San Martín et al. 1998). La mayoría de los hábitats están cercanos a cursos de agua o sobre suelos con drenaje restringido, aunque algunas subpoblaciones se encuentran en laderas boscosas secas (San Martín et al. 1998, Le Quesne et al. 2000).

Debido a la sustitución de su hábitat costero por especies introducidas, a su utilización como planta ornamental por lo llamativo de sus flores de color rojo, al uso de su tallo como materia prima para cestería y algunos aspectos ecofisiológicos como la germinación casi nula de sus semillas en invernadero y hábitat natural (Hauenstein 2002, Hechenleitner et al. 2005, Smith-Ramírez et al. 2005), es de vital importancia realizar estudios para establecer métodos de propagación que nos permitan repoblar su hábitat natural. Una forma de mejorar el estado de conservación de estas poblaciones es incrementando el recurso por medio del manejo (Santelices \& Cabello 2006). En consecuencia, es necesario desarrollar técnicas orientadas a la repoblación de áreas afectadas y una primera etapa debiera estar dirigida a conocer las diferentes formas de propagación de estas especies (Santelices 2005).

La propagación vegetativa o asexual surge como una alternativa de producción de plantas con el mismo genotipo de la planta madre, ya que la estaca posee yemas con aptitud potencial para desarrollar nuevos vástagos (Hartmann et al. 2002). Utilizando estacas de la especie a reproducir sólo es necesario que un nuevo sistema de raíces adventicias se desarrolle. No obstante, las estacas reaccionan de distinta forma de acuerdo a las especies y a factores tales como: edad de la estaca, grado de lignificación de la rama, presencia de hojas, aplicación de reguladores del crecimiento y época en que se recolectan, entre otros. Para tener éxito en el enraizamiento adventicio también se debe tener en consideración las condiciones ambientales como la humedad relativa, temperatura, luz y sustrato (Taiz \& Zeiger 2006, Escobar 2007).

La aplicación de reguladores de crecimiento, específicamente auxinas, se manifiesta en la velocidad y tasa de arraigamiento (Santelices \& Bobadilla 1997). Uno de los aspectos más estudiados en la propagación por estacas es el efecto que tienen las auxinas sobre el enraizamiento. Es por ello que el enraizamiento de $B$. corallina a partir de estacas tratadas con ácido 3-indolbutírico (AIB) resulta importante, dado que permitiría aumentar el número de individuos de la especie, ayudando a restablecer la composición y estructura de las comunidades naturales.

Por lo expuesto anteriormente, en el presente estudio se evaluó el efecto de diferentes concentraciones de AIB y la época de muestreo sobre la capacidad de enraizamiento de estacas de $B$. corallina. Se espera obtener porcentajes de enraizamiento superiores al $90 \%$ y una supervivencia y arraigamiento de las plantas de un $90 \%$.

\section{MATERIALES Y MÉTODOS}

\section{Material Vegetal}

Las estacas utilizadas en el ensayo se recolectaron de individuos adultos de $B$. corallina, en el sector de Trongol Bajo, Parcela 8 (Curanilahue $37^{\circ} 28^{\prime} \mathrm{S}$; 73⒉' O, provincia de Arauco, Chile), durante tres épocas del año (verano, otoño e invierno). El estudio se estableció en el invernadero de la Facultad de Ciencias Forestales, Universidad de Concepción, Chile. Se seleccionaron estacas de $8-10 \mathrm{~cm}$ de longitud y 2,0-2,5 mm de diámetro provenientes de madera semidura y blanda, se desinfectaron en una solución de fungicida (Benomilo y Captan 1,5 $\mathrm{gL}^{-1}$ ) durante una hora. Transcurrido este período se procedió a cortar las hojas a nivel del pecíolo dejando sólo dos hojas en el extremo superior, las que fueron cortadas a la mitad. Posteriormente se sometieron a una inmersión rápida (cinco segundos) en diferentes concentraciones de AIB (ppm): $\mathrm{T}_{1} 500, \mathrm{~T}_{2}$ 1.500, $\mathrm{T}_{3} 3.000$, $\mathrm{T}_{4} 5.000$, más el tratamiento $\mathrm{T}_{0}$ control. Una vez inducidas se establecieron en bandejas con sustrato de corteza de pino compostada y perlita (80\%:20\%) y se mantuvieron en el invernadero bajo las siguientes condiciones ambientales: $90 \%$ HR hasta la fase de rizogénesis, la humedad se mantuvo mediante riego automatizado a través de un sistema de aspersores conocido en el mercado nacional como "microjet", aplicando diferentes frecuencias de riego de acuerdo a la época del año. En época de calor se aplicaron 4 riegos diarios de una duración de 5 min cada uno, mientras 
que en el periodo frío se utilizaron 2 ó 3 riegos diarios de 2,5 min cada uno; $17-22{ }^{\circ} \mathrm{C}$ temperatura del sustrato; $22-27{ }^{\circ} \mathrm{C}$ temperatura ambiental.

En todos los ensayos las estacas permanecieron durante 5 meses en invernadero y posteriormente fueron trasladadas a vivero.

\section{ANÁLISIS DE DATOS}

Se aplicó un diseño experimental completamente aleatorio con arreglo factorial. Para el factor época y dosis de AIB, la unidad experimental estuvo constituida por 10 estacas con ocho repeticiones por tratamiento.

Después de 5 meses de establecido el ensayo se evaluó el porcentaje de supervivencia y porcentaje de enraizamiento adventicio. Además se cuantificó el potencial de crecimiento radicular mediante el número de raíces por estaca y longitud de las raíces $(\mathrm{cm})$.

La variable longitud de raíces se analizó por medio de un análisis de varianza (ANOVA) y test de Tukey para establecer diferencias entre promedios de efectos significativos. Para esto se utilizó el procedimiento GLM de SAS. Para las variables número de raíces y ausencia/ presencia de raíces se realizó un análisis de desvianza (ANDEVI), considerando la metodología de Modelos Lineales Generalizados (Mora et al. 2008). Para el número de raíces se consideró una distribución binomial negativa, debido a sobredispersión encontrada al utilizar la distribución de Poisson, con función de ligación logarítmica. Para la presencia/ausencia de raíces, la distribución usada correspondió a binomial con función de ligación Logit (Rodovalho et al. 2008). La metodología de modelos lineales generalizados se implementó en el procedimiento GENMOD de SAS, con test de contrastes para establecer diferencias entre promedios de fuentes de variación significativas.

\section{RESULTADOS}

En función de las dosis de AIB utilizadas sobre la presencia o ausencia de raíces los resultados indican que no existen diferencias significativas, sin embargo, al evaluar las variables longitud y número de raíces en función con la época de muestreo y la interacción tratamiento-época del año, existen diferencias significativas (Tabla I). Por otra parte, se observó que existe una elevada tasa de supervivencia (mayor de 95\%) en los tratamientos aplicados a las estacas recolectadas en las épocas de otoño e invierno, a diferencia de lo encontrado en las estacas recolectadas en verano donde el porcentaje fue significativamente inferior (menor de 45\%).

A pesar de que las concentraciones de AIB no afectaron significativamente la capacidad de enraizamiento de las estacas de B. corallina, la interacción entre la concentración de AIB y la época de cosecha sí mostró efecto significativo $(P<0,05)$ para el número de raíces (Tabla I). La época de cosecha fue significativa para las tres variables estudiadas (Tabla I), siendo las épocas de otoño e invierno las más significativas para obtener un mayor porcentaje de enraizamiento ( 89 y $93 \%$, respectivamente) y elongación de raíces (Tabla II). Los valores promedios encontrados para longitud de raíces, en esta procedencia, fueron de 4,7 y 4,8 $\mathrm{cm}$ para las épocas de otoño e invierno, respectivamente.

En el análisis de la interacción época-tratamiento para la variable número de raíces, se encontró que el tratamiento $T_{1}$ presentó la mejor respuesta con valores promedio de 7,9 y 8,1 raíces, en invierno y otoño, respectivamente (Tabla III).

Por otro lado, no se observó diferencias en cuanto a época y dosis hormonal en las estacas de primer orden como en las de segundo orden (datos no mostrados), ambas fueron capaces de enraizar no existiendo diferencias visuales notorias entre ellas.

TABLA I. Resultados del análisis de varianza para la longitud de raíces, y análisis de desvianza para el número de raíces (distribución binomial negativa) y presencia/ausencia de raíces (distribución binomial).

TABLE I. Analysis of results in the variance in root length, the deviance in the number of roots (negative binomial distribution), and the presence/absence of roots (binomial distribution).

\begin{tabular}{|c|c|c|c|c|c|c|c|}
\hline \multirow{2}{*}{ FuENTE DE VARIACIÓN } & \multirow{2}{*}{ GL } & \multicolumn{2}{|c|}{ LONGITUD DE RAÍCES } & \multicolumn{2}{|c|}{ NÚMERO DE RAÍCES } & \multicolumn{2}{|c|}{ Presencia de Raíces } \\
\hline & & $\mathrm{F}$ & $\mathrm{P}>\mathrm{F}$ & $\chi^{2}$ & $\mathrm{P}>\chi^{2}$ & $\chi^{2}$ & $\mathrm{P}>\chi^{2}$ \\
\hline Época (E) & 2 & 106.33 & $0.0001 * *$ & 219.09 & $0.0001 * *$ & 284.93 & $0.0001 * *$ \\
\hline Tratamiento (T) & 4 & 1.40 & $0.2321 \mathrm{~ns}$ & 4.66 & $0.324 \mathrm{~ns}$ & 6.85 & $0.1443 \mathrm{~ns}$ \\
\hline Interacción (E x T) & 8 & 0.64 & $0.7435 \mathrm{~ns}$ & 20.87 & $0.0075 * *$ & 9.67 & $0.2890 \mathrm{~ns}$ \\
\hline
\end{tabular}

** Estadísticamente significativo a $1 \%$; ns: no significativo; P: probabilidad; $\chi^{2}$ : Chi-cuadrado; F: Test F de Snedecor. / Statistically significant at $1 \%$, ns: not significant, P: probability; $\chi^{2:}$ Chi-square, F: Snedecor's F Test. 
TABla II. Promedios de potencial de crecimiento a través de longitud de raíces y presencia/ausencia de raíces por época.

TABLE II. Average growth potential of cutting as a reflection of root length and presence/absence of roots over time.

\begin{tabular}{lcccc}
\hline \multirow{2}{*}{ ÉPoca } & \multicolumn{2}{c}{ Longitud de Raíces (CM) } & \multicolumn{2}{c}{$\begin{array}{c}\text { Presencia De Raíces (escala } \\
\text { BINOMial) }\end{array}$} \\
\cline { 2 - 5 } & $\mathrm{N}$ & Promedio** & $\mathrm{N}$ & Promedio** \\
\hline Verano & 154 & $2.46 \mathrm{~b}$ & 349 & $0.44 \mathrm{~b}$ \\
Invierno & 284 & $4.80 \mathrm{a}$ & 400 & $0.93 \mathrm{a}$ \\
Otoño & 268 & $4.74 \mathrm{a}$ & 400 & $0.89 \mathrm{a}$ \\
\hline Total & 706 & 4.27 & 1149 & 0.77 \\
\hline
\end{tabular}

$\mathrm{N}$ : número de explantes medidos; ** Letras distintas en la misma columna indican que los promedios son estadísticamente diferentes (probabilidad de 99\%). / Number of explants measured; ** Different letters in the same column indicate that the averages are statistically different (probability of $99 \%$ ).

TABLA III. Fraccionamiento de la interacción Época-Tratamiento para la variable número de raíces.

TABLE III. Splitting of time-treatment interaction for the variable number of roots.

\begin{tabular}{cccc}
\hline \multirow{2}{*}{ Tratamiento } & \multicolumn{3}{c}{ Número de Raíces** } \\
\cline { 2 - 4 } & Verano & InVierno & Otoño \\
\hline $\mathrm{T}_{0}$ & $2.3 \mathrm{a} \mathrm{B}$ & $5.8 \mathrm{~b} \mathrm{~A}$ & $7.0 \mathrm{bc} \mathrm{A}$ \\
$\mathrm{T}_{1}$ & $2.5 \mathrm{a} \mathrm{B}$ & $7.9 \mathrm{a} \mathrm{A}$ & $8.1 \mathrm{a} \mathrm{A}$ \\
$\mathrm{T}_{2}$ & $2.0 \mathrm{a} \mathrm{B}$ & $6.3 \mathrm{~b} \mathrm{~A}$ & $7.7 \mathrm{ab} \mathrm{A}$ \\
$\mathrm{T}_{3}$ & $2.8 \mathrm{a} \mathrm{B}$ & $6.3 \mathrm{~b} \mathrm{~A}$ & $6.8 \mathrm{bc} \mathrm{A}$ \\
$\mathrm{T}_{4}$ & $3.5 \mathrm{a} \mathrm{B}$ & $6.1 \mathrm{~b} \mathrm{~A}$ & $6.0 \mathrm{c} \mathrm{A}$ \\
\hline
\end{tabular}

** Letras minúsculas distintas en la misma columna indican que los promedios son estadísticamente diferentes entre tratamientos dentro de una determinada época; y letras mayúsculas distintas en la misma fila indican que los promedios difieren entre épocas dentro de un determinado tratamiento (probabilidad de 99\%). $\mathrm{T}_{0}$ : tratamiento control, $\mathrm{T}_{1}$ : ácido 3-indolbutírico 500 ppm, $\mathrm{T}_{2}$ : ácido 3-indolbutírico 1.500 ppm, $\mathrm{T}_{3}$ : ácido 3-indolbutírico $3.000 \mathrm{ppm}, \mathrm{T}_{4}$ : ácido 3-indolbutírico $5.000 \mathrm{ppm}$. / Different lowercase letters in the same column indicate that the averages are statistically different between treatments within a certain time, and different capital letters in the same row indicate that the averages differ between seasons within a given treatment ( $99 \%$ probability). $\mathrm{T}_{0}$ : control treatment, $\mathrm{T}_{1}: 500 \mathrm{ppm}$ indole-3-butyric acid, $\mathrm{T}_{2}: 1,500 \mathrm{ppm}$ indole-3-butyric acid, $\mathrm{T}_{3}: 3,000 \mathrm{ppm}$ indole-3-butyric acid, $\mathrm{T}_{4}: 5,000 \mathrm{ppm}$ indole-3-butyric acid.

\section{DISCUSIÓN}

La capacidad de las auxinas para promover el desarrollo de raíces adventicias es muy conocida y se debe a que esta fitohormona aumenta el transporte de carbohidratos a la base del corte (Hartmann et al. 2002). La homogeneidad de los resultados obtenidos a los cinco meses de establecidos los ensayos difieren a los reportados para otras especies (leñosas y herbáceas), en el sentido de que la aplicación de AIB es un procedimiento que influye en el enraizamiento (Krisantini et al. 2003, Santelices \& Cabello 2006, BettiolNeto et al. 2006). Sin embargo, otros estudios concuerdan con los resultados obtenidos en esta investigación, donde las auxinas sintéticas y sus diferentes concentraciones no tuvieron un efecto significativo sobre las variables estudiadas (Hernández et al. 2005, Ruiz et al. 2005, Latsague et al.
2009). Lo anterior se puede atribuir a que posiblemente las auxinas exógenas no tuvieron un reconocimiento por parte de las células o proteína receptora para originar algún cambio metabólico, o bien la concentración interna de fitohormona fue la suficiente para inducir raíces adventicias, lo que concuerda con lo señalado por Hartmann et al. (2002).

Por otra parte, la literatura señala que AIB no es tóxico en un amplio rango de concentraciones (Hartmann et al. 2002). Esto se aprecia en este estudio, porque al aumentar la concentración de auxina no se produjo disminución en el desarrollo radicular. Esto puede indicar que la dosis óptima de AIB depende de la especie y de la condición fisiológica en que se encuentra la estaca al momento de ser recolectada.

En general, la capacidad para enraizar está influenciada por la planta madre o donadora de las estacas cosechadas (Santelices 2005) y por la época de colecta de las mismas 
(Weaver 1987). En este estudio, las estacas recolectadas en otoño e invierno obtuvieron niveles significativos en porcentajes de enraizamiento ( 89 y $93 \%$ respectivamente), en comparación con los obtenidos en verano (44\%), lo que nos señalaría que dichas épocas son las más adecuadas para recolectar material de B. corallina procedente del sector Trongol Bajo (Región del Bío-Bío). Mientras que los resultados obtenidos por Latsague et al. (2008) en plantas procedentes del sector Villa Las Araucarias, Región de la Araucanía, sólo están referidos a la época de verano y cuyas estacas presentan mayores porcentajes de enraizamiento en verano y con valores mayores de longitud y número de raíces. Sin embargo, el presente trabajo señala como factor determinante la época de muestreo sobre la dosis hormonal para la obtención de raíces adventicias, lo que puede estar influenciado por el genotipo, sumado a la condición fisiológica de las estacas al momento de las cosechas.

Estudios realizados en Nothofagus glauca Espinosa, Juglans cinerea L. y Grevillea muestran que estacas cosechadas a fines de primavera obtuvieron un alto porcentaje de enraizamiento (Krisantini et al. 2003, Pijut \& Moore 2003, Santelices \& Cabello 2006). Los resultados indican que el comportamiento de producción de raíces adventicias es propio para cada especie y puede estar determinado también por factores ambientales como luz, temperatura, fotoperíodo y estado fisiológico de la planta (Hartmann et al. 2002), estos factores estarían influenciando los cambios endógenos a nivel hormonal (Blakesley et al. 1991). En este contexto, Melgares de Aguilar et al. (2005) indican que las diferencias entre las distintas épocas se pueden atribuir a la variación de las condiciones fisiológicas internas de las estacas.

Los resultados obtenidos respecto al tipo de estaca coinciden con lo reportado por Ruiz et al. (2005) en Gmelina arborea Roxb., cuyas estacas obtenidas de la parte intermedia de la rama tuvieron un porcentaje similar al de las estacas apicales, lo que indica que el potencial de enraizamiento se mantiene en los primeros entrenudos de una rama (datos no mostrados).

Se concluye que es posible enraizar estacas semileñosas de Berberidopsis corallina, donde la capacidad de enraizamiento a niveles aceptables es independiente de la concentración de AIB utilizada, dado que no incide en el prendimiento de estacas, por lo que el testigo o control muestra valores de prendimiento altos y en algunos casos superiores que los restantes tratamientos. Por otro lado, otoño e invierno son las épocas de muestreo en que se logró un mayor porcentaje de enraizamiento y longitud de las raíces, independiente de la dosis auxínica.

La selección de las plantas madres y época de cosecha de las estacas se debe considerar en el futuro como un factor fundamental, sobre todo si se piensa realizar algún tipo de mejoramiento genético utilizando esta técnica.

\section{AGRADECIMIENTOS}

Los autores agradecen al proyecto DIUC N²03.412.0031.0 de la Dirección de Investigación de la Universidad de Concepción por el financiamiento de este estudio, y a la empresa Bosques Arauco S. A. por la facilidades otorgadas durante el trabajo de campo.

\section{BIBLIOGRAFÍA}

Alarcón, D., C. Smith-Ramírez, P. Hechenleitner, P. Ramírez de Arellano, M. Oliva, M. Pinto. 2007. Nuevas poblaciones de Berberidopsis corallina Hook.f. (Berberidopsidales: Berberidopsidaceae) en la Región del Bío-Bío, Chile: Ubicación y conservación de su hábitat. Gayana Botánica 64(2): 217-231.

Benoit, C. 1989. Libro rojo de la flora terrestre de Chile. Santiago, Chile. Ministerio de Agricultura. 157 pp.

Bettiol-Neto, J.E., R. Pio, S.C.S. Bueno, D.C.Bastos \& J.A. Scarpare-Filho. 2006. Enraizamento de estacas dos portaenxertos araticum-de-terra-fria (Rollinia sp.) e araticummirim (Rollinia emarginata Schltdl.) para Anonáceas. Ciència e Agrotecnologia 30(6): 1077-1082.

Blakesley, D., G.D. Weston \& M.C. Elliot. 1991. Endogenous levels of indole-3-acetic acid and abcisic acid during the rooting of Cotinus coggyria cuttings taking at diferent times of the year. Plant Growth Regulation 10: 1-12.

Escobar, R. 2007. Manual de viverización. Eucalyptus globulus a raíz cubierta. Trama Impresores S.A. Chile. 229 pp.

Hartmann, H.T., D.E. Kester, F.T. Davies \& R.L. Geneve. 2002. Plant propagation: Principles and Practice. 7th ed. Prentice Hall Inc. New Jersey, USA. 880 pp.

Hauenstein, E. 2002. Notas sobre Berberidopsis corallina Hooker (Berberidopsidaceae) ¿Especie en Peligro?. Gestión Ambiental (Chile) 8: 63-69.

Hechenleitner, V.P., M.F. Gardner, P.I. Thomas, C. Echeverría, B. Escobar, P. Brownless \& C. Martínez. 2005. Plantas amenazadas del centro-sur de Chile. Distribución, conservación y propagación. Primera Edición. Valdivia, Chile. Universidad Austral de Chile y Real Jardín Botánico de Edimburgo. 188 pp.

Hernández, J.R., H. Aramendia \& C.E. Cardona. 2005. Influencia del ácido indolbutírico y ácido naftalenacético sobre el enraizamiento de esquejes de caña flecha (Gynerium sagittatum Aubl.). Temas Agrarios 10: 5-13.

Krisantini, S., M. Johnston, R.R. Williams \& C. Beveridge. 2003. Propagation of Grevillea. Combined Proceeding. The International Plant Propagators' Society 53: 154-158.

Latsague, M., P. Sáez \& E. Hauenstein. 2008. Inducción de enraizamiento en estacas de Berberidopsis corallina con ácido indolbutírico. Bosque 29(3): 227-230.

Latsague, M., P. SÁez \& J. Yáñez. 2009. Efecto del ácido indolbutírico en la capacidad rizogénica de estacas de Eucryphia glutinosa. Bosque 30(2): 102-105.

Le Quesne, C., R. Carrasco \& L. Sandoval. 2000. Lista de puntos florísticos de algunas especies en peligro, raras y vulnerables en la Región del Bío-Bío. CONAF Serie Técnica Año 3(4): $1-16$. 
Melgares de Aguilar, J., D. González, A. Navarro, S. Bañón \& F. GARCíA. 2005. Influencia de la estacionalidad en el enraizamiento de esquejes de Coriaria myrtifolia L. Actas de Horticultura, V Congresso Ibérico de Ciências Hortícolas p. 457-461.

Mora, F., L.M. Goncalves, C.A. Scapim, E.N. Martins \& M.F.P.S. Machado. 2008. Generalized lineal models for the analysis of binary data from propagation experiments of Brazilian orchids. Brazilian Archives of Biology and Technology 51(5): 963-970.

Pisut, P.M. \& M.J. Moore. 2003. Early season softwood cuttings effective for vegetative propagation of Juglans cinerea. Hortscience 37: 697-700.

Rodovalho, M.A., F. Mora, E.M. Santos, C.A. Scapim \& E. ARNHOLD. 2008. Survival heritability in 169 families of white grain popcorn: A Bayesian approach. Ciencia e Investigación Agraria 35(3): 303-309.

Ruiz, R., J.J. Vargas, V.M. Cetina \& A. Villegas. 2005. Efecto del ácido indolbutírico (AIB) y tipo de estaca en el enraizado de Gmelina arborea Roxb. Revista Fitotecnia Mexicana. 28(4): 319-326.

San Martín, J., T. Yáñez \& J. Loyola. 1998. Plantas trepadoras.
Universidad de Talca. Consultado el 25 de abril 2010. Disponible en http://arbol.tripod.cl/3.htm.

SANTElices, R. 2005. Efecto del árbol madre sobre la rizogénesis de Nothofagus alessandrii. Bosque 26(3): 133-136.

Santelices, R. \& A. Bobadilla. 1997. Arraigamiento de estacas de Quillaja saponaria Mol. y Peumus boldus Mol. Bosque 18(2): 77-85.

SAntelices, R. \& A. CABello. 2006. Efecto del ácido indolbutírico, del tipo de la cama de arraigamiento, del substrato, y del árbol madre en la capacidad de arraigamiento de estacas de Nothofagus glauca (Phil.) Krasser. Revista Chilena de Historia Natural 79: 55-64.

Smith-Ramírez, C., B. Campillo, J.L. Celis-Diez \& M. GARDNER. 2005. Historia natural de la enredadera endémica Berberidopsis corallina. En: C. Smith-Ramírez, J. Armesto \& C. Valdovinos (eds.), Historia, Biodiversidad y Ecología de los Bosques Costeros de Chile. pp. 284-288. Editorial Universitaria, Santiago, Chile.

TAiz, L. \& E. ZeIGER. 2006. Plant physiology. 4th ed. Massachusetts: Sinauer Associates. 705 pp.

Weaver, R. 1987. Reguladores de crecimiento de las plantas en la agricultura. Editorial Trillas, México. 622 pp.

Recibido: 30.03.11

Aceptado: 10.06.11 